\title{
NEW RESULTS ON THE MINIMAL POLYNOMIAL FOR LINEAR RECURRING SEQUENCES OVER $\mathbb{F}_{q^{m}}$
}

\section{YONGSHENG TANG and HEQIAN XU}

Department of Mathematics

Hefei Normal University

Hefei 230601, Anhui

P. R. China

e-mail: ysh_tang@163.com

heqianxu@sina.com

\begin{abstract}
Let $S^{(t)}=\left(S_{1}, S_{2}, \ldots, S_{t}\right)$ be a linear recurring sequence over $\mathbb{F}_{q^{m}}$, where each $S_{i}$ is a linear recurring sequence over $\mathbb{F}_{q} m_{i}$ with minimal polynomial $f_{i}(x)$ over $\mathbb{F}_{q^{m_{i}}}$ and the finite field $\mathbb{F}_{q^{m_{i}}}$ is a subfield of finite field $\mathbb{F}_{q^{m}}$. Let $T$ be a linear transformation of $\mathbb{F}_{q^{m}}$ over $\mathbb{F}_{q}$. Denote $T\left(S^{(t)}\right)=\left(T_{1}\left(S_{1}\right), T_{2}\left(S_{2}\right), \ldots, T_{t}\left(S_{t}\right)\right)$, where $T_{i}$ is a linear transformation of $\mathbb{F}_{q^{m}}$ over $\mathbb{F}_{q^{m_{i}}}$. In this paper, we study the minimal polynomial and linear complexity of linear recurring sequence $S^{(t)}$. If the canonical factorization of each $f_{i}(x)$ in $\mathbb{F}_{q^{m}}[x]$ is known, we determine the minimal polynomial and
\end{abstract}

2010 Mathematics Subject Classification: 94B55, 94A60, 11B50.

Keywords and phrases: linear recurring sequences, linear complexity, minimal polynomial. This research is supported by the Natural Science Foundation of Anhui Province (No. 1808085MA15), Key University Science Research Project of Anhui Province (No. KJ2018A0497), and Foundation for Anhui Province College Outstanding Young Talents Program (gxyqZD2016228).

Received November 15, 2018

(C) 2019 Scientific Advances Publishers 
linear complexity of linear recurring sequence $S^{(t)}$ over $\mathbb{F}_{q^{m}}$. Furthermore, we determine the minimal polynomial of $T\left(S^{(t)}\right)$ if the minimal polynomial of each $S_{i}$ without multiple roots is known.

\section{Introduction}

The concept of the linear complexity is very useful in the study of the security of stream ciphers (see [1, 3, 13]). In a stream cipher, a key stream sequence $S$ must have large linear complexity $L$ because one can use the Berlekamp-Massey algorithm to generate the whole sequence by observing $2 L$ consecutive elements of $S$ (see [6]).

Recently, motivated by the study of vectorized stream cipher systems, the joint linear complexity and joint minimal polynomial of multisequences have been investigated (see [7-11]). Wang studied the multisequence shift-register synthesis with applications to decoding cyclic codes in [14].

The notation of linear complexity over $\mathbb{F}_{q}$ of linear recurring sequences over $\mathbb{F}_{q^{m}}$ was introduced by Ding et al. in [1]. Meidl and Özbudak in [8] studied the linear complexity over $\mathbb{F}_{q}$ and over $\mathbb{F}_{q^{m}}$ for linear recurring sequences. Gao and $\mathrm{Fu}$ in [11] studied the minimal polynomial and linear complexity over $\mathbb{F}_{q}$ of a linear recurring sequence $S$ over $\mathbb{F}_{q^{m}}$ with minimal polynomial $h(x)$ over $\mathbb{F}_{q^{m}}$, then discussed the minimal polynomial of sequence obtained from the componentwise linear transformation of a linear recurring sequence in [12].

Let $S_{1}, S_{2}, \ldots, S_{t}$ be linear recurring sequences over $\mathbb{F}_{q}$ with minimal polynomial $h_{1}(x), h_{2}(x), \ldots, h_{t}(x)$, respectively. If $h_{1}(x), h_{2}(x)$, $\ldots, h_{t}(x)$ are pairwise relatively prime, then the minimal polynomial of the linear sequence $S=\left(S_{1}, S_{2}, \ldots, S_{t}\right)$ is given by the product of $h_{1}(x), h_{2}(x), \ldots, h_{t}(x)$. This is the fact of Theorem 8.57 in [5]. However, 
not much work has been done on the minimal polynomials of the linear sequence $S=\left(S_{1}, S_{2}, \ldots, S_{t}\right)$ over $\mathbb{F}_{q^{m}}$ and $T\left(S^{(t)}\right)=\left(T_{1}\left(S_{1}\right), T_{2}\left(S_{2}\right)\right.$, $\left.\ldots, T_{t}\left(S_{t}\right)\right)$, where $S_{i}$ is a linear recurring sequence over $\mathbb{F}_{q^{m_{i}}}, \mathbb{F}_{q^{m_{i}}}$ is a subfield of $\mathbb{F}_{q^{m}}, T$ is a linear transformation of $\mathbb{F}_{q^{m}}$ over $\mathbb{F}_{q}$, and $T_{i}$ is a linear transformation of $\mathbb{F}_{q^{m}}$ over $\mathbb{F}_{q^{m_{i}}}$.

In this paper, we study the minimal polynomial and linear complexity of linear recurring sequence $S^{(t)}=\left(S_{1}, S_{2}, \ldots, S_{t}\right)$, where $S_{i}$ is a linear recurring sequence in $\mathbb{F}_{q^{m_{i}}}$ with minimal polynomial $f_{i}(x)$ and $\mathbb{F}_{q^{m_{i}}}$ is a subfield of $\mathbb{F}_{q^{m}}$. If the canonical factorization of each $f_{i}(x)$ of $S_{i}$ in $\mathbb{F}_{q^{m}}[x]$ is known, we determine the minimal polynomial and linear complexity of linear recurring sequence $S^{(t)}$ over $\mathbb{F}_{q^{m}}$. Furthermore, let $T$ be a linear transformation of $\mathbb{F}_{q^{m}}$ over $\mathbb{F}_{q}$. Denote $T\left(S^{(t)}\right)=\left(T_{1}\left(S_{1}\right), T_{2}\left(S_{2}\right), \ldots, T_{t}\left(S_{t}\right)\right)$, where $\quad T_{i} \quad$ is a linear transformation of $\mathbb{F}_{q}$ over $\mathbb{F}_{q^{m_{i}}}$. We determine the minimal polynomial of $T\left(S^{(t)}\right)$ if the minimal polynomial of each $S_{i}$ without multiple roots is known.

\section{Preliminaries}

Let $S=\left(s_{0}, s_{1}, s_{2}, \ldots\right)$ be a sequence with terms in the finite field $\mathbb{F}_{q^{m}}$ is called a linear recurring sequence over $\mathbb{F}_{q^{m}}$ with characteristic polynomial

$$
f(x)=c_{0}+c_{1} x+\cdots+c_{n-1} x^{n-1}+x^{n} \in \mathbb{F}_{q^{m}}[x],
$$


if for all $k \geq 0$,

$$
c_{0} s_{k}+c_{1} s_{k+1}+\cdots+c_{n-1} s_{k+n-1}+s_{k+n}=0 .
$$

The minimal polynomial of a linear recurring sequence $S$ is defined to be the uniquely determined characteristic polynomial over $\mathbb{F}_{q^{m}}$ of $S$ with least degree. The linear complexity over $\mathbb{F}_{q^{m}}$ of $S$ is the degree of the minimal polynomial over $\mathbb{F}_{q^{m}}$, denoted by $L_{\mathbb{F}_{q}}(S)$. If $h(x)$ is the minimal polynomial over $\mathbb{F}_{q^{m}}$ of $S$, then $h(x)$ divides the characteristic polynomial $f(x)$ of $S$. Furthermore, the sequence $S=\left(s_{0}, s_{1}, s_{2}, \ldots\right)$ over $\mathbb{F}_{q^{m}}$ can be viewed as an element of $\mathbb{F}_{q^{m}}((x))$ :

$$
S(x)=\sum_{i=0}^{\infty} s_{i} x^{-i}
$$

which is called the generating function associated with $S$, where $\mathbb{F}_{q^{m}}((x))$ is the field of formal Laurent series over $\mathbb{F}_{q^{m}}$. In [10], the sequence $S=\left(s_{0}, s_{1}, s_{2}, \ldots\right)$ over $\mathbb{F}_{q^{m}}$ is a linear recurring sequence with minimal polynomial $h(x)$ if and only if $S(x)=\frac{g(x)}{h(x)}$, with $g(x) \in \mathbb{F}_{q^{m}}[x]$, $\operatorname{deg}(g(x))<\operatorname{deg}(h(x))$, and $\operatorname{gcd}(g(x), h(x))=1$.

The mapping $\sigma$ from $\mathbb{F}_{q^{m}}$ to $\mathbb{F}_{q^{m}}$ is defined as follows:

$$
\sigma: \alpha \mapsto \alpha^{q}
$$

It is obvious that $\sigma$ is a field automorphism of $\mathbb{F}_{q^{m}}$. We know that $\sigma(a)=a$ if and only if $a \in \mathbb{F}_{q}$. A field automorphism of $\mathbb{F}_{q^{m}}$ which leaves every element of $\mathbb{F}_{q}$ fixed is called a field automorphism of $\mathbb{F}_{q^{m}}$ over $\mathbb{F}_{q}$. In particular, $\sigma$ is a field automorphism of $\mathbb{F}_{q^{m}}$ over $\mathbb{F}_{q}$, we 
call $\sigma$ the Frobenius automorphism of $\mathbb{F}_{q^{m}}$ over $\mathbb{F}_{q}$. Then we can extend $\sigma$ to be a ring automorphism of the polynomial ring $\mathbb{F}_{q^{m}}[x]$ as follows:

For $f(x)=a_{0}+a_{1} x+\cdots+a_{n} x^{n} \in \mathbb{F}_{q^{m}}[x]$,

$$
\sigma: f(x) \mapsto \sigma(f(x))
$$

where $\sigma(f(x))=a_{0}^{q}+a_{1}^{q} x+\cdots+a_{n}^{q} x^{n}$. It is easy to prove that $\sigma(f(x))$ is irreducible over $\mathbb{F}_{q^{m}}$ if and only if $f(x)$ is irreducible over $\mathbb{F}_{q^{m}}$. In this paper, for any $f(x), g(x) \in \mathbb{F}_{q^{m}}[x]$, we will use the following fact that:

$$
\sigma(f(x) g(x))=\sigma(f(x)) \sigma(g(x)) \text {, }
$$

and if $g(x) \neq 0$, we have

$$
\sigma\left(\frac{f(x)}{g(x)}\right)=\frac{\sigma(f(x))}{\sigma(g(x))}
$$

Denote $\sigma^{0}$ the identity mapping and $\sigma^{i}$ the $i$-th usual composition of $\sigma$. Denote $k(f)$ the minimum positive integer $k$ such that $\sigma^{k}(f(x))=f(x)$. The set of automorphism of $\mathbb{F}_{q^{m}}$ over $\mathbb{F}_{q}$ forms a group with respect to the composition of mappings, which is called the Galois group of $\mathbb{F}_{q^{m}}$ over $\mathbb{F}_{q}$ and denoted by $\operatorname{Gal}\left(\mathbb{F}_{q^{m}} / \mathbb{F}_{q}\right)$. Now, we give the following lemma on Galois groups.

Lemma 1 ([15]). Gal $\left(\mathbb{F}_{q^{m}} / \mathbb{F}_{q}\right)=\langle\sigma\rangle$. More precisely, $\sigma^{0}=1, \sigma^{1}=\sigma$, $\ldots, \sigma^{m-1}$ are all the automorphism of $\mathbb{F}_{q^{m}}$ over $\mathbb{F}_{q}$. Furthermore, let $d$ be a positive divisor of $m$. Then $\sigma^{d}$ is the Frobenius automorphism of $\mathbb{F}_{q^{m}}$ over $\mathbb{F}_{q^{d}}$ and $\operatorname{Gal}\left(\mathbb{F}_{q^{m}} / \mathbb{F}_{q^{d}}\right)=\left\langle\sigma^{d}\right\rangle$ is a cyclic group of order $m / d$, denoted by $\left|\left\langle\sigma^{d}\right\rangle\right|$. 


\section{Main Results}

In the finite field $\mathbb{F}_{q^{m}}$, let $m=p_{1}^{e_{1}} p_{2}^{e_{2}} \cdots p_{t}^{e_{t}}$, where the $p_{i}$ are relatively prime and $e_{i}$ are positive integers, for $i=1,2, \ldots, t$. Denote $m_{i}=p_{i}^{e_{i}}$ and $d_{i}=m / m_{i}$. Then $m_{1}, m_{2}, \cdots, m_{t}$ are the $t$ distinct positive divisors of $m$. In the sequel, we will use the following lemma.

Lemma 2 ([4]). Let $N_{1}, N_{2}, \ldots, N_{m}$ be all normal subgroups of the group G. If $G=\left\langle\bigcup_{i=1}^{m} N_{i}\right\rangle$, and for each $i, N_{k} \bigcap\left\langle\bigcup_{i \neq k} N_{i}\right\rangle=\langle e\rangle$, then $G \cong \prod_{i=1}^{m} N_{i}$

Now, we give the following proposition for analyzing the minimal polynomial of linear recurring sequence $S^{(t)}$ over $\mathbb{F}_{q^{m}}$.

Proposition 1. Let $\mathbb{F}_{q^{m}}$ be a finite field, $\sigma$ be a field automorphism of $\mathbb{F}_{q^{m}}$ over $\mathbb{F}_{q}$ and $\mathbb{F}_{q^{m_{i}}} \subseteq \mathbb{F}_{q^{m}}$, for $i=1,2, \ldots, t$. Then

$$
\langle\sigma\rangle \cong\left\langle\sigma^{d_{1}}\right\rangle \times\left\langle\sigma^{d_{2}}\right\rangle \times \cdots \times\left\langle\sigma^{d_{t}}\right\rangle
$$

Proof. Firstly, $\left\langle\sigma^{d_{1}}\right\rangle,\left\langle\sigma^{d_{2}}\right\rangle, \ldots,\left\langle\sigma^{d_{t}}\right\rangle$ are all normal subgroups of the cyclic group $\langle\sigma\rangle$ since they are all cyclic subgroups of the cyclic group $\langle\sigma\rangle$. Secondly, by Lemma 1, $|\langle\sigma\rangle|=\left|\left\langle\sigma^{d_{1}}\right\rangle\right|\left|\left\langle\sigma^{d_{2}}\right\rangle\right| \cdots\left|\left\langle\sigma^{d_{t}}\right\rangle\right|$. On the other hand, $\left\langle\bigcup_{i=1}^{t}\left\langle\sigma^{d_{i}}\right\rangle\right\rangle \subseteq\langle\sigma\rangle$. Hence

$$
\left\langle\bigcup_{i=1}^{t}\left\langle\sigma^{d_{i}}\right\rangle\right\rangle=\langle\sigma\rangle .
$$


Furthermore, for $1 \leq i \leq t,\left\langle\sigma^{d_{i}}\right\rangle \bigcap\left\langle\bigcup_{j \neq i}\left\langle\sigma^{d_{j}}\right\rangle\right\rangle=\{1\}$. Suppose on the contrary that there exists $\sigma^{j}$ such that $\sigma^{j} \in\left\langle\sigma^{d_{i}}\right\rangle$ and $\sigma^{j} \in\left\langle\sigma^{d_{c}}\right\rangle$, where $\left\langle\sigma^{d_{c}}\right\rangle \in\left\langle\bigcup_{j \neq i}\left\langle\sigma^{d_{j}}\right\rangle\right\rangle$. Then the order of $\sigma^{j}$ divides $m / d_{i}$ and also divides $m / d_{c}$. But $m / d_{i}$ and $m / d_{c}$ are relatively prime. Hence $j=0$. By Lemma 2, we obtain

$$
\langle\sigma\rangle \cong\left\langle\sigma^{d_{1}}\right\rangle \times\left\langle\sigma^{d_{2}}\right\rangle \times \cdots \times\left\langle\sigma^{d_{t}}\right\rangle
$$

which completes the proof.

For details of groups, we refer the reader to Hungerford [4] and Wan [15]. From Proposition 1, we define

$$
\Phi:\langle\sigma\rangle \rightarrow\left\langle\sigma^{d_{1}}\right\rangle \times\left\langle\sigma^{d_{2}}\right\rangle \times \cdots \times\left\langle\sigma^{d_{t}}\right\rangle,
$$

by

$$
\Phi\left(\sigma^{i}(P(x))\right)=\left(\sigma^{d_{1} i}(P(x)), \sigma^{d_{2} i}(P(x)), \ldots, \sigma^{d_{t} i}(P(x))\right) .
$$

Then $\sigma^{d_{1} i}(P(x)), \sigma^{d_{2} i}(P(x)), \ldots, \sigma^{d_{t} i}(P(x))$ are in $\mathbb{F}_{q^{m_{1}}}[x], \mathbb{F}_{q^{m_{2}}}[x], \ldots$, $\mathbb{F}_{q^{m_{t}}}[x]$, respectively.

Definition 1 ([11]). The canonical factorization of a monic irreducible polynomial $f_{i}(x)$ over $\mathbb{F}_{q^{m_{i}}} \subseteq \mathbb{F}_{q^{m}}$ and $n_{i}=\operatorname{deg}\left(f_{i}(x)\right)$ into monic irreducibles over $\mathbb{F}_{q^{m}}$ is defined by

$$
f_{i}(x)=P_{i}(x) \sigma^{d_{i}}\left(P_{i}(x)\right) \cdots \sigma^{d_{i}(u-1)}\left(P_{i}(x)\right),
$$

where $u=\operatorname{gcd}\left(n_{i}, m\right)$ and $P_{i}(x)$ is a monic irreducible polynomial over $\mathbb{F}_{q^{m}}$. 
Theorem 1. Let each $S_{i}$ be a linear recurring sequence over $\mathbb{F}_{q^{m_{i}}} \subseteq$ $\mathbb{F}_{q^{m}}$ with minimal polynomial $f_{i}$, for $i=1,2, \ldots, t$. Suppose that the canonical factorization of $f_{i}$ into monic irreducibles over $\mathbb{F}_{q^{m}}$ is given by

$$
f_{i}=P_{i} \sigma^{d_{i}}\left(P_{i}\right) \cdots \sigma^{d_{i}\left(u_{i}-1\right)}\left(P_{i}\right),
$$

where $u_{i}=\operatorname{gcd}\left(n_{i}, m\right)$. Denote $n_{i} / u_{i}=k$ and $u=\max \left\{u_{1}, u_{2}, \ldots, u_{t}\right\}$. Then $S^{(t)}=\left(S_{1}, S_{2}, \ldots, S_{t}\right)$ with minimal polynomial $h(x)$ over $\mathbb{F}_{q^{m}}$ is of the following form:

$$
\begin{aligned}
h(x)= & \Phi^{-1}\left(P_{1}, P_{2}, \ldots, P_{t}\right) \Phi^{-1}\left(\sigma^{d_{1}}\left(P_{1}\right), \sigma^{d_{2}}\left(P_{2}\right), \ldots, \sigma^{d_{t}}\left(P_{t}\right)\right) \\
& \cdots \Phi^{-1}\left(\sigma^{d_{1}(u-1)}\left(P_{1}\right), \sigma^{d_{2}(u-1)}\left(P_{2}\right), \ldots, \sigma^{d_{t}(u-1)}\left(P_{t}\right)\right) .
\end{aligned}
$$

Proof. Since $n_{i} / u_{i}=k$, then there exists an isomorphism $\Phi$ satisfying Equation (1). Let $\Phi^{-1}\left(P_{1}, P_{2}, \ldots, P_{t}\right)=P \in \mathbb{F}_{q^{m}}[x]$. Then

$$
h(x)=P(x) \sigma(P) \cdots \sigma^{u-1}(P) .
$$

We claim that $P, \sigma(P), \ldots, \sigma^{u-1}(P)$ are distinct irreducible polynomials. Assume that some $\sigma^{i}(P), 0 \leq i \leq u-1$ is not irreducible. Then there exist two nonconstant polynomials $Q_{1}, Q_{2}$ in $\mathbb{F}_{q^{m}}[x]$ such that $\sigma^{i}(P)=Q_{1} Q_{2}$. Therefore, $\Phi\left(\sigma^{i}(P)\right)=\Phi\left(Q_{1}\right) \Phi\left(Q_{2}\right)$. By the definition of $\Phi$, we obtain

$$
\Phi\left(\sigma^{i}(P)\right)=\left(\sigma^{d_{1} i}\left(P_{1}\right), \sigma^{d_{2} i}\left(P_{2}\right), \ldots, \sigma^{d_{t} i}\left(P_{t}\right)\right) .
$$

Let $\Phi\left(Q_{1}\right)=\left(Q_{1,1}, Q_{1,2}, \ldots, Q_{1, t}\right)$ and $\Phi\left(Q_{2}\right)=\left(Q_{2,1}, Q_{2,2}, \ldots, Q_{2, t}\right)$, where $Q_{i, j}$ are nonconstant polynomials over $\mathbb{F}_{q^{m_{j}}}[x]$ for $1 \leq i \leq 2$, $1 \leq j \leq t$. Hence, for $1 \leq j \leq t$, 


$$
\sigma^{d_{j} i}\left(P_{j}\right)=Q_{1, j} Q_{2, j}
$$

which contradicts to the fact that $\sigma^{d_{j} i}\left(P_{j}\right)$ is irreducible over $\mathbb{F}_{q} m_{j}$.

Furthermore, suppose that there exist $\sigma^{i}(P), \sigma^{j}(P), 0 \leq i \neq j \leq u-1$ such that $\sigma^{i}(P)=\sigma^{j}(P)$. Then, by the definition of $\Phi$, we get

$$
\left(\sigma^{d_{1} i}\left(P_{1}\right), \sigma^{d_{2} i}\left(P_{2}\right), \ldots, \sigma^{d_{t} i}\left(P_{t}\right)\right)=\left(\sigma^{d_{1} j}\left(P_{1}\right), \sigma^{d_{2} j}\left(P_{2}\right), \ldots, \sigma^{d_{t} j}\left(P_{t}\right)\right)
$$

Therefore, for $1 \leq k \leq t$,

$$
\sigma^{d_{k} i}\left(P_{k}\right)=\sigma^{d_{k} j}\left(P_{k}\right)
$$

However, by the definition of $u$, there exists $u_{s}$ such that $u_{s}=u$. This gives a contradiction since $\sigma^{d_{s} i}\left(P_{s}\right), \sigma^{d_{s} j}\left(P_{s}\right)$ are relatively prime.

On the other hand,

$$
\begin{aligned}
& \Phi(P)=\left(P_{1}, P_{2}, \ldots, P_{t}\right), \\
& \Phi(\sigma(P))=\left(\sigma^{d_{1}}\left(P_{1}\right), \sigma^{d_{2}}\left(P_{2}\right), \ldots, \sigma^{d_{t}}\left(P_{t}\right)\right), \\
& \quad \vdots \\
& \Phi\left(\sigma^{u-1}(P)\right)=\left(\sigma^{d_{1}(u-1)}\left(P_{1}\right), \sigma^{d_{2}(u-1)}\left(P_{2}\right), \ldots, \sigma^{d_{t}(u-1)}\left(P_{t}\right)\right) .
\end{aligned}
$$

Denote $g_{i}=P_{i} \sigma^{d_{i}}\left(P_{i}\right) \cdots \sigma^{d_{i}(u-1)}\left(P_{i}\right)$. Then $f_{i}$ must divide $g_{i}$ in $\mathbb{F}_{q^{m}}[x]$. Hence, $g_{i}$ is a characteristic polynomial over $\mathbb{F}_{q^{m}}$ of $S_{i}$. Then $h(x)$ is a characteristic polynomial over $\mathbb{F}_{q^{m}}$ of $S^{(t)}$. Let $H(x)$ be the minimal polynomial over $\mathbb{F}_{q^{m}}$ of $S^{(t)}$. Then $H(x)$ divides $h(x)$ in $\mathbb{F}_{q^{m}}[x]$. Since $P, \sigma(P), \ldots, \sigma^{u-1}(P)$ are distinct irreducible polynomials, then we assume, 
without loss of generality, that there exist $i, j$ and $0 \leq i \leq j \leq u-1$ such that $H(x)=\sigma^{i}(P) \sigma^{i+1}(P) \cdots \sigma^{j}(P)$. By the definition of $\Phi$, we get

$$
\begin{aligned}
& \Phi\left(\sigma^{i}(P)\right)=\left(\sigma^{d_{1} i}\left(P_{1}\right), \sigma^{d_{2} i}\left(P_{2}\right), \ldots, \sigma^{d_{t} i}\left(P_{t}\right)\right), \\
& \Phi\left(\sigma^{i+1}(P)\right)=\left(\sigma^{d_{1}(i+1)}\left(P_{1}\right), \sigma^{d_{2}(i+1)}\left(P_{2}\right), \ldots, \sigma^{d_{t}(i+1)}\left(P_{t}\right)\right), \\
& \quad \vdots \\
& \Phi\left(\sigma^{j}(P)\right)=\left(\sigma^{d_{1} j}\left(P_{1}\right), \sigma^{d_{2} j}\left(P_{2}\right), \ldots, \sigma^{d_{t} j}\left(P_{t}\right)\right) .
\end{aligned}
$$

By the definition of $u$, there exists $u_{s}$ such that $u_{s}=u$. Therefore

$$
\sigma^{d_{s} i}\left(P_{s}\right) \sigma^{d_{s}(i+1)}\left(P_{s}\right) \cdots \sigma^{d_{s} j}\left(P_{s}\right)
$$

is the minimal polynomial over $\mathbb{F}_{q^{m_{s}}}$ of $S_{s}$. This contradicts to the fact that $f_{s}=P_{s} \sigma^{d_{s}}\left(P_{s}\right) \cdots \sigma^{d_{s}(u-1)}\left(P_{s}\right)$ is the minimal polynomial over $\mathbb{F}_{q^{m_{s}}}$ of $S_{s}$. Therefore, we have

$$
H(x)=P \sigma(P) \cdots \sigma^{u-1}(P),
$$

which completes the proof.

Remark. If $m=p^{e}$, where $p$ is a prime and $e$ is a positive integer, then Theorem 1 can be viewed as a necessary condition of Proposition 2.1 in [8] or Theorem 6 in [11].

Corollary 1. With the notation of Theorem 1, the linear complexity over $\mathbb{F}_{q^{m}}$ of $S^{(t)}$ is given by

$$
L_{\mathbb{F}_{q^{m}}}\left(S^{(t)}\right)=u k
$$

The following example illustrates the above results. 
Example. Let $\mathbb{F}_{2^{2}} \subseteq \mathbb{F}_{2^{6}}$, where $\mathbb{F}_{2^{6}}$ is defined by $\gamma^{6}+\gamma+1=0$ and $\mathbb{F}_{2^{2}}$ is defined by $\alpha^{2}+\alpha+1=0$. Then $\mathbb{F}_{2^{2}}=\left\{0,1, \alpha, \alpha^{2}=1+\alpha\right\}$. Let $S_{1}$ be a periodic sequence over $\mathbb{F}_{2^{2}}$ with the least period 3 . The first period terms of $S_{1}$ are given by

$$
0,1, \alpha^{2}
$$

The minimal polynomial over $\mathbb{F}_{2^{2}}$ of $S_{1}$ is $x^{2}+(1+\alpha) x+\alpha$. The canonical factorization of $x^{2}+(1+\alpha) x+\alpha$ into monic irreducibles over $\mathbb{F}_{2^{6}}$ is given by:

$$
x^{2}+(1+\alpha) x+\alpha=(x+\alpha) \sigma^{3}(x+\alpha) .
$$

Let $\mathbb{F}_{2^{3}} \subseteq \mathbb{F}_{2^{6}}$ and let $\beta$ be a root of $x^{3}+x+1$ in $\mathbb{F}_{2^{3}}$. Let $S_{2}$ be a periodic sequence over $\mathbb{F}_{2^{3}}$ with the least period 21 . The first period term of $S_{2}$ are given by

$$
1,0,1,1,1,0,0,1,0,1,1,1,0,0,1,0,1,1,1,0,0 .
$$

The minimal polynomial over $\mathbb{F}_{2^{3}}$ of $S_{2}$ is $x^{3}+x+1$. The canonical factorization of $x^{3}+x^{2}+1$ into monic irreducibles over $\mathbb{F}_{2^{6}}$ is given by:

$$
x^{3}+x^{2}+1=(x+\beta) \sigma^{2}(x+\beta) \sigma^{4}(x+\beta) .
$$

Then $S^{(2)}=\left(S_{1}, S_{2}\right)$ can be seen the linear sequence over $\mathbb{F}_{2^{6}}$. By Theorem 1, the minimal polynomial of $S^{(2)}$ is

$$
\begin{aligned}
h(x) & =\Phi^{-1}(x+\alpha, x+\beta) \Phi^{-1}\left(\sigma^{3}(x+\alpha) \cdot \sigma^{2}(x+\beta)\right) \Phi^{-1}\left(\sigma^{6}(x+\alpha), \sigma^{4}(x+\beta)\right), \\
& =\Phi^{-1}(x+\alpha, x+\beta) \Phi^{-1}\left(x+1, x+\beta^{2}\right) \Phi^{-1}\left(x+\alpha, x+\beta^{4}\right) .
\end{aligned}
$$


Therefore

$$
L_{\mathbb{F}_{2} 6}\left(S^{(2)}\right)=3
$$

In the following, we generalize the approach suggested in [12] for the linear recurring sequence $S$ over $\mathbb{F}_{q^{m}}$ to the linear recurring sequence $S^{(t)}$ over $\mathbb{F}_{q^{m}}$. We determine the minimal polynomial of $T\left(S^{(t)}\right)=\left(T_{1}\left(S_{1}\right), T_{2}\left(S_{2}\right), \ldots, T_{t}\left(S_{t}\right)\right)$, where $T$ is a linear transformation of $\mathbb{F}_{q^{m}}$ over $\mathbb{F}_{q}, T_{i}$ is a linear transformation of $\mathbb{F}_{q^{m}}$ over $\mathbb{F}_{q^{m}}$, and the minimal polynomial of each $S_{i}$ without multiple roots is known. First, we introduce some definitions.

Definition 2 ([12]). The linear transformation $T_{i}$ of $\mathbb{F}_{q^{m}}$ over $\mathbb{F}_{q^{m_{i}}}$ is defined as

$$
T_{i}(x)=c_{i, 0}+c_{i, 1} x^{q}+\cdots+c_{i, d_{i}-1} x^{q^{d_{i}-1}}
$$

where $d_{i}=m / m_{i}$ and $c_{i, 0}, c_{i, 1}, \ldots, c_{i, d_{i}-1}$ are unique in $\mathbb{F}_{q^{m}}$.

From Definition 2, the linear transformation $T$ of $\mathbb{F}_{q^{m}}$ over $\mathbb{F}_{q}$ is defined as

$$
T\left(S^{(t)}\right)=\left(T_{1}\left(S_{1}\right), T_{2}\left(S_{2}\right), \ldots, T_{t}\left(S_{t}\right)\right)
$$

where $T_{i}$ is a linear transformation of $\mathbb{F}_{q^{m}}$ over $\mathbb{F}_{q^{m_{i}}}$. Let $k_{i}$ be a positive factor of $m_{i}$ and $m_{i}=k_{i} t_{i}$. Then $T_{k_{i}, j}(x)$ denote the following polynomial:

$$
T_{k_{i}, j}(x)=c_{i, j} x^{q^{j}}+c_{i, k_{i}+j} x^{q^{k_{i}+j}}+\cdots+c_{i,\left(t_{i}-1\right) k_{i}+j} x^{q^{\left(t_{i}-1\right) k_{i}+j}}, 0 \leq j<k_{i} .
$$


For any positive integer $n$, the mapping $\mu_{i}$ is defined in [12] as follows:

$$
\begin{aligned}
& \left.\mu_{i}: \bigcup_{n=1}^{\infty}\left(\mathbb{F}_{q}\right)^{n}\right)^{n} \rightarrow \mathbb{Z}, \\
& \left(a_{i, 1}, a_{i, 2}, \ldots, a_{i, n}\right) \mapsto \mu_{i}\left(a_{i, 1}, a_{i, 2}, \ldots, a_{i, n}\right),
\end{aligned}
$$

where

$$
\mu_{i}\left(a_{i, 1}, a_{i, 2}, \ldots, a_{i, n}\right)= \begin{cases}0 & \text { if }\left(a_{i, 1}, a_{i, 2}, \ldots, a_{i, n}\right)=(0,0, \ldots, 0) \\ 1 & \text { otherwise }\end{cases}
$$

Define a mapping $\mu$ as follows:

$\mu: \bigcup_{i=1}^{t}\left(\bigcup_{n=1}^{\infty}\left(\mathbb{F}_{q} m_{i}\right)^{n}\right) \rightarrow \mathbb{Z}$

$\left(a_{1,1}, \ldots, a_{1, n}, \ldots, a_{t, 1}, \ldots, a_{t, n}\right) \mapsto\left(\mu_{1}\left(a_{1,1}, \ldots, a_{1, n}\right), \ldots, \mu_{t}\left(a_{t, 1}, \ldots, a_{t, n}\right)\right)$,

where $a_{i, j} \in \mathbb{F}_{q^{m_{i}}}, 1 \leq i \leq t, 1 \leq j \leq n$, if each $\mu_{i}\left(a_{i, 1}, \ldots, a_{i, n}\right)=0$, then $\mu\left(a_{1,1}, \ldots, a_{1, n}, \ldots, a_{t, 1}, \ldots, a_{t, n}\right)=0 ; \quad$ otherwise $\mu\left(a_{1,1}, \ldots, a_{1, n}, \ldots\right.$, $\left.a_{t, 1}, \ldots, a_{t, n}\right)=1$.

Lemma 3 ([12]). Let $S$ be a linear recurring sequence over $\mathbb{F}_{q^{m}}$ with irreducible minimal polynomial $h(x)$. Assume that $g(x)=b_{0}+b_{1} x+\cdots$ $+b_{l} x^{l}$ is the polynomial over $\mathbb{F}_{q^{m}}$ such that $S(x)=g(x) / h(x)$ and $l<\operatorname{deg}(h(x))$. Let $T$ be a linear transformation of $\mathbb{F}_{q^{m}}$ over $\mathbb{F}_{q}$ given by Definition 2. Then the minimal polynomial of $T(S)$ is given by

$$
h(x)^{e_{0}}(\sigma(h(x)))^{e_{1}} \cdots\left(\sigma^{(k(h)-1)}(h(x))\right)^{e_{k(h)-1}},
$$

where $e_{j}=\mu\left(T_{k(h), j}\left(b_{0}\right), T_{k(h), j}\left(b_{1}\right), \ldots, T_{k(h), j}\left(b_{l}\right)\right)$ for $0 \leq j<k(h)$, and $T_{k, j}$ is defined by (3). 
From Theorem 1 and Lemma 3, we directly get the following theorem.

Theorem 2. Let each $S_{i}$ be a linear recurring sequence over $\mathbb{F}_{q^{m_{i}}} \subseteq$ $\mathbb{F}_{q^{m}}$ with irreducible minimal polynomial $h_{i}(x)$ and $n=\operatorname{deg}\left(h_{i}(x)\right)$. Suppose that $g_{i}(x)=b_{i_{0}}+b_{i_{1}} x+\cdots+b_{i_{l}} x^{i_{l}}$ is the polynomial over $\mathbb{F}_{q^{m_{i}}}$ such that $S_{i}(x)=g_{i}(x) / h_{i}(x)$ and $\max \left\{l_{1}, l_{2}, \ldots, l_{t}\right\}<\operatorname{deg}\left(h_{1}(x)\right)=\operatorname{deg}$ $\left(h_{2}(x)\right)=\cdots=\operatorname{deg}\left(h_{t}(x)\right)$. Let $T$ be a linear transformation of $\mathbb{F}_{q^{m}}$ over $\mathbb{F}_{q}$ given by (2). Then the minimal polynomial of $T\left(S^{(t)}\right)$ is of the following form:

$$
\begin{aligned}
& \Phi^{-1}\left(h_{1}, h_{2}, \ldots, h_{t}\right)^{e_{0}} \Phi^{-1}\left(\sigma^{d_{1}}\left(h_{1}\right), \sigma^{d_{2}}\left(h_{2}\right), \ldots, \sigma^{d_{t}}\left(h_{t}\right)\right)^{e_{1}} \\
& \cdots \Phi^{-1}\left(\sigma^{d_{1}(u-1)}\left(h_{1}\right), \sigma^{d_{2}(u-1)}\left(h_{2}\right), \ldots, \sigma^{d_{t}(u-1)}\left(h_{t}\right)\right)^{e_{u}-1}
\end{aligned}
$$

where $u=\operatorname{gcd}(n, m)$ and $e_{j}=\mu\left(T_{u, j}\left(b_{1_{0}}\right), \ldots, T_{u, j}\left(b_{t_{0}}\right), \ldots, T_{u, j}\left(b_{1_{l}}\right)\right.$, $\left.\ldots, T_{u, j}\left(b_{t_{l}}\right)\right)$ for $0 \leq j<u$.

\section{Conclusion}

In this paper, we study the minimal polynomial and linear complexity of linear recurring sequence $S^{(t)}=\left(S_{1}, S_{2}, \ldots, S_{t}\right)$, where each $S_{i}$ is a linear recurring sequence over finite field $\mathbb{F}_{q^{m_{i}}}$ with minimal polynomial $f_{i}(x)$ over $\mathbb{F}_{q^{m_{i}}}$ and the finite field $\mathbb{F}_{q^{m_{i}}}$ is a subfield of finite field $\mathbb{F}_{q^{m}}$. If the canonical factorization of each $f_{i}(x)$ in $\mathbb{F}_{q^{m}}[x]$ is known, we determine the minimal polynomial and linear complexity of linear recurring sequence $S^{(t)}$ over $\mathbb{F}_{q^{m}}$. Furthermore, let $T$ be a linear transformation of $\mathbb{F}_{q^{m}}$ over $\mathbb{F}_{q}$. Denote $T\left(S^{(t)}\right)=\left(T_{1}\left(S_{1}\right)\right.$, 
$\left.T_{2}\left(S_{2}\right), \ldots, T_{t}\left(S_{t}\right)\right)$, where $T_{i}$ is a linear transformation of $\mathbb{F}_{q^{m}}$ over $\mathbb{F}_{q} m_{i}$. We determine the minimal polynomial of $T\left(S^{(t)}\right)$ if the minimal polynomial of each $S_{i}$ without multiple roots is known. It would be interesting to investigate the minimal polynomial of linear recurring sequence $S^{(t)}=\left(S_{1}, S_{2}, \ldots, S_{t}\right)$ over $\mathbb{F}_{q}$, with the condition that the degrees of the canonical factorization of each $f_{i}(x)$ into monic irreducibles over $\mathbb{F}_{q^{m}}$ are different, which is also a natural open problem.

\section{References}

[1] C. Ding, G. Xiao and W. Shan, The Stability Theory of Stream Ciphers, Lecture Notes in Computer Science, Springer, Berlin, 1991.

[2] S. W. Golomb, Shift Register Sequences, Aegean Park Press, Laguna Hills, CA, 1982.

[3] S. W. Golomb and G. Gong, Signal Design for Good Correlation for Wireless Communication, Cryptography and Radar, Cambridge University Press, Cambridge, UK, 2005.

[4] T. W. Hungerford, Algebra, New York, Springer-Verlag, 1974.

[5] R. Lidl and H. Niederreiter, Finite Fields, Cambridge University Press, Cambridge, 1997.

[6] J. Massey, Shift register synthesis and BCH decoding, IEEE Transactions on Information Theory 15(1) (1969), 122-127.

DOI: https://doi.org/10.1109/TIT.1969.1054260

[7] W. Meidl and H. Niederreiter, The expected value of the joint linear complexity of periodic multisequences, Journal of Complexity 19(1) (2003), 61-72.

DOI: https://doi.org/10.1016/S0885-064X(02)00009-2

[8] W. Meidl and F. Özbudak, Linear complexity over $\mathbb{F}_{q}$ and over $\mathbb{F}_{q^{m}}$ for linear recurring sequences, Finite Fields and their Applications 15(1) (2009), 110-124.

DOI: https://doi.org/10.1016/j.ffa.2008.09.004

[9] W. Meidl and F. Özbudak, Generalized Joint Linear Complexity of Linear Recurring Multisequences, In: S. W. Golomb et al. (editors), Sequences and their Applications SETA 2008, In: Lecture Notes in Computer Science, Springer (LNCS), Berlin, 5203 (2008), 266-277. 
[10] H. Niederreiter, Sequences with Almost Perfect Linear Complexity Profile, in: D. Chaum and W. L. Price (editors), Advances in Cryptology-EUROCRYPT 1987, In: LNCS, Springer, Berlin, 304 (1988), 37-51.

[11] Z. H. Gao and F. W. Fu, The minimal polynomial over $\mathbb{F}_{q}$ of linear recurring sequence over $\mathbb{F}_{q^{m}}$, Finite Fields and their Applications 15(6) (2009), 774-784.

DOI: https://doi.org/10.1016/j.ffa.2009.07.002

[12] Z. H. Gao and F. W. Fu, The minimal polynomial of a sequence obtained from the componentwise linear transformation of a linear recurring sequence, Theoretical Computer Science 411(44-46) (2010), 3883-3893.

DOI: https://doi.org/10.1016/j.tcs.2010.07.014

[13] R. A. Rueppel, Analysis and Design of Stream Ciphers, New York, Springer-Verlag, 1986.

[14] L. P. Wang, The Vector Key Equation and Multisequence Shift Register Synthesis, In: M. Fossorier et al. (editors), Applied Algebra, Algebraic Algorithms and ErrorCorrecting Codes, Lecture Notes in Computer Science, Springer, Berlin, 3857 (2006), 68-75.

[15] Z. X. Wan, Lectures on Finite Fields and Galois Rings, World Scientific Publish. Co., Singapore, 2003. 